\title{
Aneurisma de artéria renal em rim transplantado: reparo ex vivo e reimplante do enxerto
}

\author{
Renal artery aneurysm in a transplanted kidney: ex vivo graft repair \\ and reimplantation
}

\section{Cesar Roberto Busato ${ }^{1}$, Carlos Alberto de Lima Utrabo ${ }^{1}$, Wilson Freire de Sousa ${ }^{2}$, Ricardo Zanetti Gomes ${ }^{1}$, Joel Kengi Hosoume ${ }^{1}$, Eliziane Hoeldtke ${ }^{1}$, Rafael Inácio Brandão ${ }^{3}$, Dieyson Martins de Melo Costa ${ }^{3}$}

\section{Resumo}

Aneurisma verdadeiro de artéria renal em rim transplantado é ocorrência rara. As possibilidades de tratamento dependem do tamanho, da localização do aneurisma e da clínica apresentada pelo paciente. Descreve-se um caso de aneurisma gigante de artéria renal em rim transplantado que recebeu tratamento ex vivo e reimplante na fossa ilíaca direita. Detalhes do procedimento cirúrgico são descritos.

Palavras-chave: Aneurisma da artéria renal, autotransplante renal, complicações em rim transplantado.

\section{Introdução}

O transplante constitui-se hoje terapêutica bem estabelecida, sendo empregado como tratamento de escolha da insuficiência renal crônica irreversível em todo o mundo. A maior sobrevida desses pacientes fez com que a expectativa de complicações vasculares por aterosclerose aumentasse ${ }^{1}$. O tratamento dessas complicações exige estratégias cirúrgicas que promovam a proteção do enxerto renal ${ }^{2}$. Estas podem ser realizadas de maneira convencional, in situ, por técnica endovascular ${ }^{3}$, ou, quando as condições anatômicas não permitirem, por nefrectomia seguida de perfusão hipotérmica, correção da patologia vascular e reimplante na fossa ilíaca ${ }^{4-7}$.

\section{Relato de caso}

Paciente masculino, com 51 anos, recebeu um transplante renal de doador vivo há 60 meses. Cirurgia realizada sem intercorrências, permanecendo assintomático

\begin{abstract}
True aneurysm of a renal artery in a transplanted kidney is a rare occurrence. Treatment options depend on size and location of the aneurysm and the patient's clinical condition. We report a case of a giant aneurysm of the renal artery in a transplanted kidney that was treated ex vivo and reimplanted in the right iliac fossa. Details of the surgical procedure are described.
\end{abstract}

Keywords: Renal artery aneurysm, renal autotransplant, complications in a transplanted kidney.

até 2 meses atrás, quando, em controle realizado, passou a apresentar aumento gradual da creatinina sérica, chegando a $2,7 \mathrm{mg} / \mathrm{dL}$, sendo submetido, então, a investigação clínica. Uma ultra-sonografia demonstrou dilatação aneurismática da artéria renal transplantada, estendendo-se do hilo à anastomose com a artéria ilíaca externa, apresentando trombo mural. Na tomografia computadorizada com contraste (Figura 1), os achados foram confirmados, e o tamanho do aneurisma, mensurado em 3,9 × 4,0 x $6,5 \mathrm{~cm}$.

\section{Cirurgia}

O implante renal foi retirado da fossa ilíaca direita e recebeu tratamento ex vivo, sendo perfundido pela veia renal com solução de Euro-Collins. Na operação de bancada, notou-se erosão do parênquima pelo aneurisma que foi ressecado. Utilizou-se a veia safena magna para

1. Serviço de Cirurgia Vascular, Departamento de Cirurgia, Santa Casa de Misericórdia de Ponta Grossa (SCMPG), Ponta Grossa, PR.

2. Serviço de Urologia, Departamento de Cirurgia, SCMPG, Ponta Grossa, PR.

3. Residente, Serviço de Cirurgia Vascular, Departamento de Cirurgia, SCMPG, Ponta Grossa, PR.

Não foram declarados conflitos de interesse associados à publicação deste artigo.

Trabalho apresentado como pôster no X Encontro Paranaense de Angiologia e Cirurgia Vascular, realizado em Curitiba, PR, em 9 e 10 de maio de 2008.

Artigo submetido em 29.07.08, aceito em 16.12.08. 


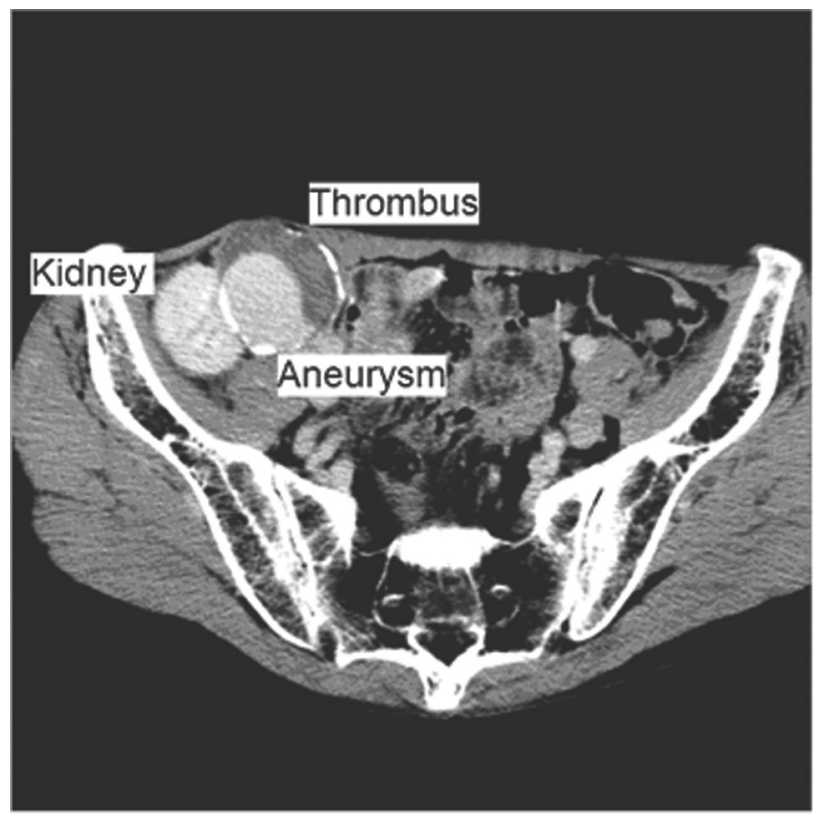

Figura 1 - Tomografia computadorizada mostrando aneurisma gigante de artéria renal com trombo mural em rim transplantado na fossa ilíaca direita

confecção de um enxerto venoso espiralado, de diâmetro semelhante à veia renal (Figura 2).

O rim foi reimplantado na fossa ilíaca direita de modo que o tubo venoso ficasse interposto entre o coto venoso do rim e a veia ilíaca comum. Um segmento de safena magna foi utilizado para a confecção da ponte entre o coto arterial do rim e a artéria ilíaca comum. A via urinária foi anastomosada de maneira términolateral ao ureter nativo (Figura 3).

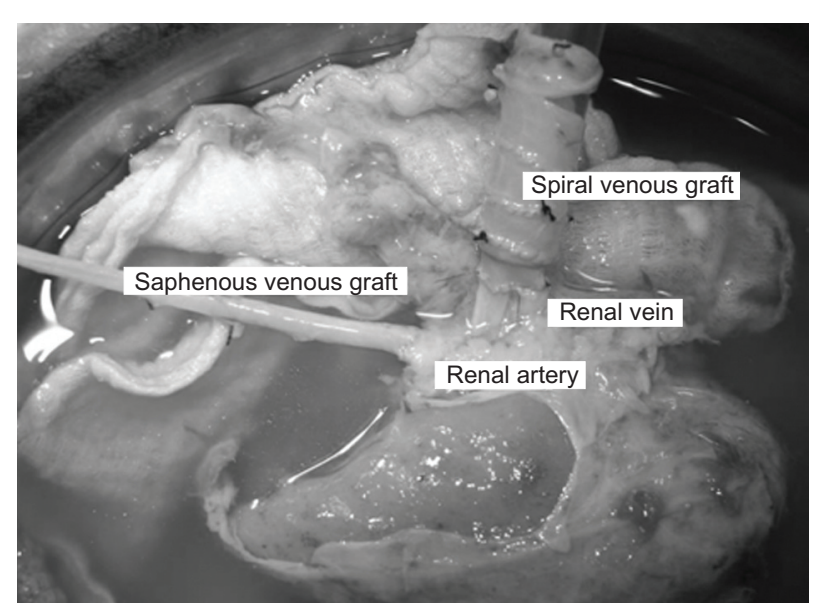

Figura 2 - Enxerto venoso espiralado e segmento de veia safena anastomosados aos vasos renais

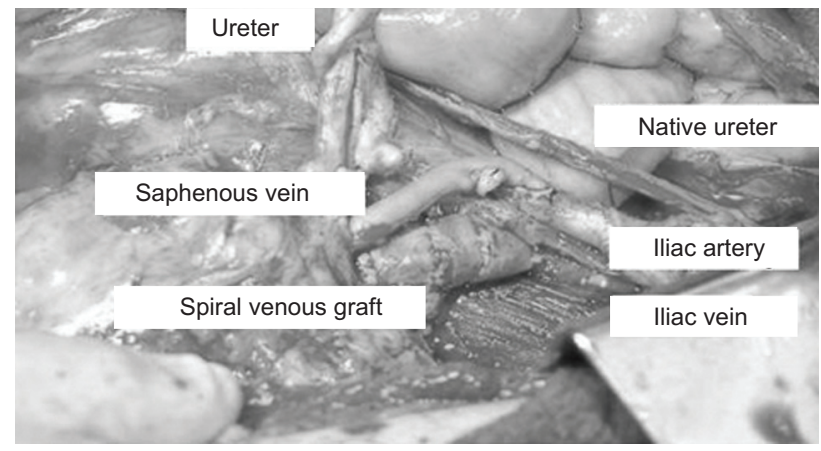

Figura 3 - Enxerto renal reimplantado aos vasos ilíacos comuns e ureter nativo na fossa ilíaca direita

No pós-operatório, o paciente evoluiu com melhora da função renal, e a creatinina diminuiu para $1,4 \mathrm{mg} / \mathrm{dL}$. Recebeu alta hospitalar sem complicações.

\section{Discussão}

As complicações dos aneurismas de artéria renal estão associadas a risco de vida por ruptura, especialmente em pacientes grávidas ${ }^{8}$, e perda do enxerto em casos de rim transplantado ${ }^{9}$. Aneurismas de mais de 2 $\mathrm{cm}$ de diâmetro ou que apresentem algum tipo de sintomatologia decorrente de sua presença têm indicação para tratamento cirúrgico ${ }^{10}$. Em 1967, Ota et al. realizaram o primeiro reparo de artéria renal, ex vivo, por hipertensão renovascular ${ }^{11}$. Em 1971, Grein et al. utilizaram a mesma técnica para correção de aneurisma de artéria renal $^{12}$. Richardson et al., em 1990, descreveram um caso de ruptura de aneurisma de artéria renal em paciente grávida com rim transplantado ${ }^{13}$. Ressecção de aneurisma em rim transplantado com reparo local foi relatada por Dunkow et al. em $1994^{14}$ e Guleria et al. em $1998^{15}$. Mais recentemente, a utilização de técnicas endovasculares tem se mostrado bastante efetiva para casos que apresentam uma anatomia favorável ${ }^{3,16}$. A necessidade de ressecção de um longo segmento da artéria comprometida pelo aneurisma e o sacrifício de parte da veia renal envolvida nesse caso, necessários para a nefrectomia, obrigaram a interpor um segmento venoso no coto arterial renal e confeccionar um enxerto espiralado para a veia. Essa condição obrigou a realizar a correção $e x$ vivo e proceder ao reimplante do enxerto renal pela segunda vez.

O reimplante renal, com reconstrução vascular extracorpórea, é uma técnica complexa com indicação nos 
casos em que o aneurisma da artéria não pode ser corrigido por técnica endovascular ou tratamento in situ. Constitui-se alternativa válida para casos selecionados.

\section{Referências}

1. Lachachi F, Ostyn E, Sekkal S, et al. Successful surgical management of a ruptured abdominal aortic aneurysm in a renal transplant patient. A case report. J Cardiovasc Surg (Torino). 1998;39:765-7.

2. Ierardi RP, Coll DP, Kumar A, Solomon BR, Kerstein MD, Matsumoto T. Abdominal aortic aneurysectomy after kidney transplantation: case report and review of the literature. Am Surg. 1996;62:961-6.

3. Cardozo MA, Lichtenfels E, Erlin Jr N, Raupp E, Tarasconi DP. Endovascular treatment of renal artery aneurysm using microcoil embolization and renal blood flow preservation: case report. J Vasc Bras. 2007;6:167-70.

4. Zhang J, Feng R, Feng X, et al. [Aneurysm repair in vitro and renal revascularization and renal autogenous transplantation for complex renal artery aneurysm in solitary kidney]. Zhonghua Wai Ke Za Zhi. 2007;45:1253-6.

5. Galmés I, Burgos FJ, Rodriguez-Luna JM, et al. ["Ex-situ" reconstructive surgery of the renal arteries]. Actas Urol Esp. 1996;20:30-6.

6. López-Fando Lavalle L, Burgos Revilla J, Sáenz Medina J, et al. [Renal autotransplantation: a valid option in the resolution of complex cases]. Arch Esp Urol. 2007;60:255-65.

7. Unno N, Yamamoto N, InuzuKa K, et al. Laparoscopic nephrectomy, ex vivo repair, and autotransplantation for a renal artery aneurysm: Report of a case. Surg Today. 2007;37:169-72.

8. Dayton B, Helgerson RB, Sollinger HW, Acher CW. Ruptured renal artery aneurysm in pregnant uninephric patient: successful ex vivo repair and autotransplantation. Surgery. 1990;107:708-11.
9. Cánovas Ivorra J, Guardiola Mas A, Nicolas Torralba JA, et al. [Dissecting aneurysm of the renal artery in patient with transplantation. Report of case. Review of the literature]. Arch Esp Urol. 2003;56:1059-62.

10. El Tayar AR, Labruzzo C, Haritopoulos K, Hakin NS. Renal artery aneurysm: ex vivo repair and autotranplantation: case report and review of the literature. Int Surg. 2003;88:61-3.

11. Ota K, Mori S, Awane Y, Ueno A. Ex situ repair of renal artery for renovascular hypertension. Arch Surg. 1967;94:370-3.

12. Glein LE, Glaes G, Gustafsson A, Storm B. Total bloodlesseness for extracorporeal organ repair. Rev Surg. 1971;28:305-16.

13. Richardson AJ, Liddington M, Jaskowski A, Murie JA, Gillmer M, Morris PJ. Pregnancy in a renal transplant recipient complicated by rupture of a transplant renal artery aneurysm. Br J Surg. 1990;77:228-9.

14. Dunkow PD, Abraham JS, Johnson RW. True aneurysm of the transplanted renal artery in a kidney transplant recipient. Nephrol Dial Transplant 1994;9:1495-7.

15. Guleria S, Ahmad N, Pollard SG, Newstead CG, Lodge PA. Transplant renal artery aneurysm following venous patch repair of a traction injury to the renal artery. Nephrol Dial Transplant 1998;13:1577-8

16. Henke PK, Cardneau JD, Welling TH 3rd, et al. Renal artery aneurysms. A 35-year clinical experience with 252 aneurysms in 168 patients. Ann Surg. 2001;234:454-62.

Correspondência

César Roberto Busato

Rua Saldanha da Gama, 425

CEP 84015-130 - Ponta Grossa, PR

Tel.: (42) 3028.4245

Fax: (42) 3224.3288

E-mail: crbusato@brturbo.com.br 